\title{
Francisco José de Caldas y Thenorio y la arquitectura en la Nueva Granada
}

\section{Artículo de Reflexión}

\section{Jesús Antonio Villalobos Rubiano}

Investigador independiente, Colombia

villalobosrubiano@gmail.com

Recibido: 27 de octubre de 2017

Aceptado: 10 de enero de 2018

Cómo citar este artículo: Villalobos Rubiano, Jesús Antonio (2018). Francisco José de Caldas y Thenorio y la arquitectura en la Nueva Granada. Calle14 revista de investigación en el campo del arte, 13 (24) pp. 344-359. DOI: https://doi.org/10.14483/21450706.13530 


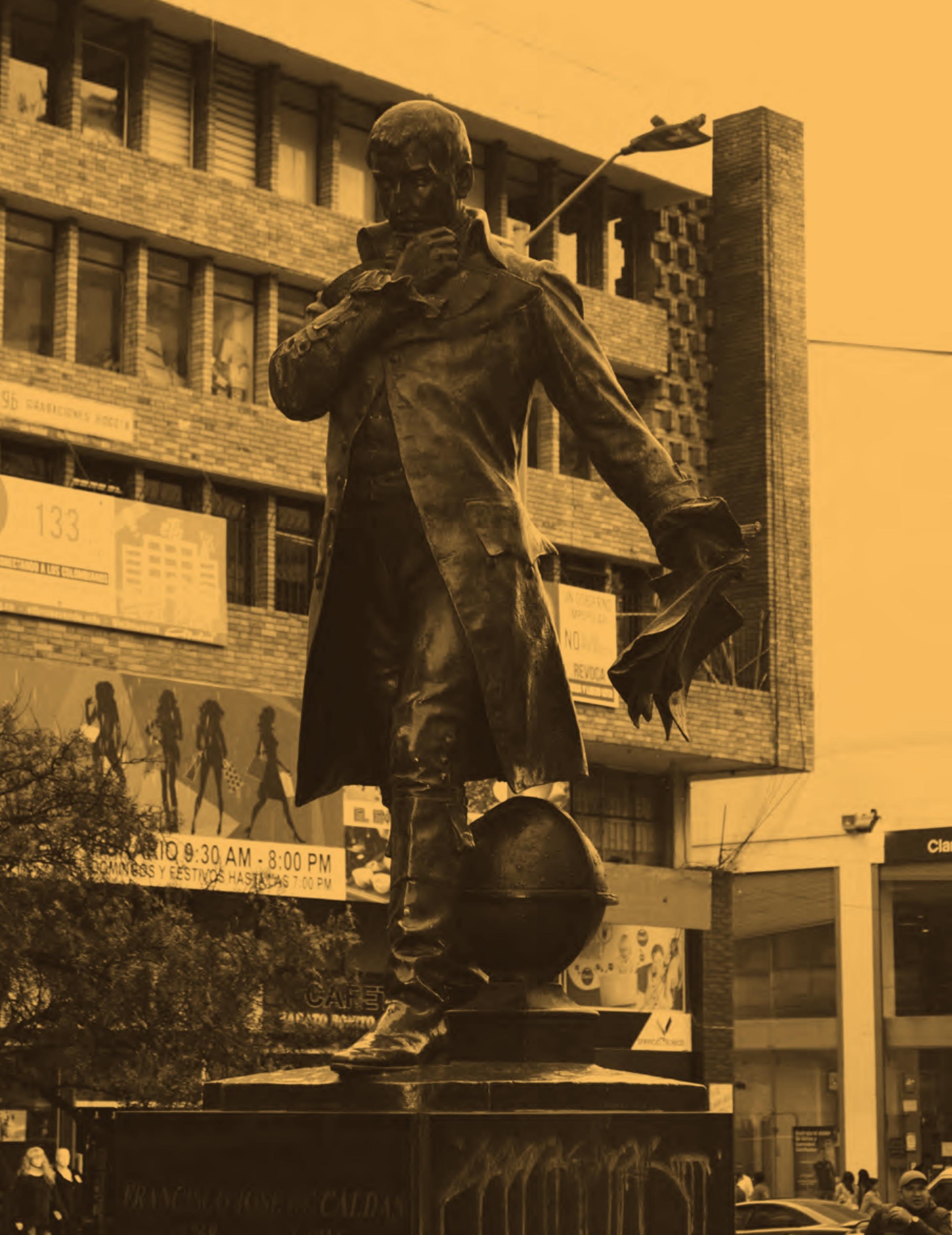




\title{
Resumen
}

El eminente científico neogranadino Francisco José de Caldas, prócer de la independencia de Colombia, se destaca por su legado científico, pero se ha dejado de lado una faceta de su vida: los aportes que realizó en el estudio, análisis, práctica y enseñanza de la arquitectura en la Nueva Granada. En el transcurso de sus viajes por el virreinato de la Nueva Granada, analizó las construcciones de las diferentes ciudades que visitó, a partir de su amplio conocimiento de Palladio y Vignola, como del estilo barroco, que para esa época se imponía. Igualmente, realiza descripciones de monumentos arqueológicos de la arquitectura inca emplazados en la Presidencia de Quito. Finalmente, como parte del ejército neogranadino, construye fortificaciones, fábricas de pólvora y cañones, y funda la primera academia militar donde se enseña por primera vez en Colombia, de manera oficial, Arquitectura.

\section{Palabras claves}

Arquitectura, Nueva Granada, Francisco José de Caldas.

\section{Francisco José de Caldas and Thenorio and Architecture in New Granada}

\begin{abstract}
The eminent neogranadino scientist Francisco José de Caldas, hero of the independence of Colombia, stands out for his scientific legacy, but a facet of his life has been left aside: the contributions he made to the study, analysis, practice and teaching of architecture in New Granada. In the course of his travels through the Viceroyalty of New Granada, he analyzed the constructions of the different cities he visited, from his extensive knowledge of Palladio and Vignola, as well as of the Baroque style, which was dominant at that time. He also makes descriptions of archaeological monuments of Inca architecture located in the Presidency of Quito. Finally, as part of the New Granada army, he builds fortifications, gunpowder factories and cannons, and founds the first military academy where Architecture is officially taught in Colombia for the first time.
\end{abstract}

\section{Keywords}

Architecture, New Granada, Francisco José de Caldas.

Francisco José de Caldas et Thenorio et l'architecture à la Nouvelle-Grenade

\section{Résumé}

L'éminent scientifique neo-grenadien Francisco Jose de Caldas, héros de l'indépendance de la Colombie, se démarque par ses contributions aux sciences, mais on a négligé une facette de sa vie : sa contribution à l'étude, l'analyse, la pratique et l'enseignement d'architecture à la NouvelleGrenade. Au cours de ses voyages autour de la vice-royauté de Nouvelle-Grenade, il a analysé les constructions des différentes villes qu'il visitait à partir de sa connaissance approfondie de Palladio et Vignola, et du style baroque qui prévalait à cette époque. Il a effectué également des descriptions de monuments archéologiques de l'architecture inca déployés dans la présidence de Quito. Enfin, dans le cadre de l'armée neo-grenadienne, il a construit des fortifications, des canons et des usines de poudre, et fonda la première académie militaire où on a enseigné officiellement I'Architecture pour la première fois en Colombie.

\section{Mots clés}

Architecture, Nouvelle-Grenade, Francisco José de Caldas. 


\section{Resumo}

O eminente cientista neogranadino Francisco José de Caldas, herói da independência da Colômbia, destaca-se a partir de seu legado científico, mas uma faceta da sua vida é ignorada: as contribuições feitas no estudo, análise, prática e ensino de arquitetura em Nova Granada. Durante suas viagens ao redor do Vice-Reino de Nova Granada, analisou as construções das diferentes cidades que ele visitou, de seu amplo conhecimento de Palladio e Vignola, e do estilo barroco que prevaleceu naquela época. Ele também faz descrições de monumentos arqueológicos da arquitetura inca localizados na Presidência de Quito. Finalmente, como parte do exército de Nova Granada, constrói fortificações, fábricas de pólvora e canhões, e funda a primeira academia militar onde a Arquitetura é oficialmente ensinada na Colômbia pela primeira vez.

\section{Palavras chaves}

Arquitetura, Nova Granada, Francisco José de Caldas.

\section{Sug runa suti Francisco José de Caldas y Thenorio kawachiku imasamka kausai Nueva Granada sutipi}

\section{Maillallachiska:}

Kai runa iacha aidachiska kawachingapa imasami ka allilla rurangapa imapas iachichiska tukuikunata kawachispa ruraspa, tapuchispa kausaskakama challaspa. Kikinpa kausaita sugsinama churaspa chimanda llukanchimi paininga Tukui nukanchimanda kawagkunata.

\section{Rimangapa Ministidukuna}

Ñugpamanda tapuchii, nueva granada kunaurramanda, Francisco José de Calda chasa suti 
La relación de Francisco José de Caldas con la Arquitectura no ha sido un tema de amplia profundización en la historia de la Arquitectura en Colombia. Los temas objeto de la investigación en la vida de Caldas están vinculados principalmente con la botánica, la astronomía, la física atmosférica, la geografía económica, la cartografía e ingeniería y demás campos en los cuales incursionó este eminente científico neogranadino.

Luego es importante, doscientos años después de su muerte, identificar la vinculación de Caldas con la arquitectura a partir de las evidencias en su obra epistolar y los datos históricos encontrados en algunos documentos.

En su obra epistolar entre 1801 y 1804, Caldas realiza descripciones de la arquitectura en la Presidencia de Quito, de la que se sabe que tuvo una amplia influencia del barroco español. En esta misma presidencia, durante sus viajes realizados por Otavalo y Ambato, se encuentra con monumentos arqueológicos de la arquitectura inca, a los cuales dedica páginas enteras para su descripción.

Posteriormente, con su arribo a la ciudad de Santafé y la labor encomendada de director del Real Observatorio Astronómico de esta ciudad, realiza una descripción del mismo y analiza sus deficiencias como observatorio en estas latitudes y finalmente, hacia 1814 , se destacan sus trabajos como ingeniero militar e instructor de la Escuela Militar de Cuerpos Facultativos, en la ciudad de Medellín.

\section{Síntesis de la arquitectura en el virreinato de la Nueva Granada a mediados del siglo XVIII e inicios del siglo XIX}

Como punto de partida, es importante establecer las influencias estilísticas de Europa en la arquitectura del virreinato de la Nueva Granada para entender la relación de Caldas con la arquitectura de su tiempo, e igualmente identificar las razones que lo vincularon con esta disciplina.

A mediados del siglo XVIII, la Compañía de Jesús estableció colegios y escuelas dentro del reordenamiento de tierras, y se fundaron nuevos poblados producto del proceso de migración de españoles a la Nueva Granada. Igualmente, en este proceso de crecimiento de las ciudades españolas en América, la Real Cédula de 1787 ordenó la creación de cementerios y obras civiles, orden que solo se comenzó a cumplir en 1791 en Santafé de Bogotá.
El virrey Ezpeleta ordenó en 1790 en la ciudad de Santafé la construcción de una alameda de un kilómetro de longitud desde San Victorino hasta la recoleta de San Diego, la denominó Alameda Vieja; otra, una alameda de tres kilómetros entre San Victorino y Puente Aranda, Alameda Nueva, y en ese mismo proceso de transformación, ordenó en 1792 la construcción del Puente del Común. Posteriormente, en 1805, el virrey Amar y Borbón le encomendó al ingeniero Domingo Esquiaqui la construcción de un camellón recto de veinte kilómetros entre la plaza de San Victorino y la alameda de San Diego.

Estas intervenciones en la pequeña ciudad de Santafé son producto de la influencia del arte barroco en España, a partir de la presencia de arquitectos italianos en el Reino, de donde se destaca la importancia de lo público y la desinteriorización de la vivienda construida en la colonia. (Arango, 1993, p.73).

En Santafé se sintió el espíritu del barroco, por cuanto la construcción de estos espacios públicos permitió que las personas disfrutaran de la calle a partir de actividades públicas. Esta situación logró que los pobladores, acostumbrados a permanecer dentro de sus casas, salieran a la vía pública y participaran de eventos culturales y políticos, lo que constituyó el abono para los posteriores hechos de emancipación ocurridos en 1810.

Las construcciones que durante la colonia estuvieron descubiertas en su fachada, durante el periodo en que el barroco llegó a América se decoraron con ornamentos exteriores y se transformaron ampliando sus ventanas puertas y balcones.

A partir de las reformas introducidas en 1766 por el rey Carlos III en España, directamente contra el monopolio de la educación por parte de la Iglesia, se tuvo como consecuencia la expulsión de la Compañía de Jesús de España y el sometimiento de la educación a la regencia de la monarquía, con la implantación de un modelo moderno de enseñanza. Dentro de estas reformas se destacó la creación del Real Jardín Botánico de Madrid, que hacía parte del proceso de liberación del comercio en América y de la necesidad de incluir nuevos productos hallados en estas tierras para su comercialización; también se destacó la intención de industrializar algunos productos tanto en España como en las colonias de ultramar.

En el virreinato de la Nueva Granada, el ilustre abogado Francisco Antonio Moreno y Escandón, nacido en 


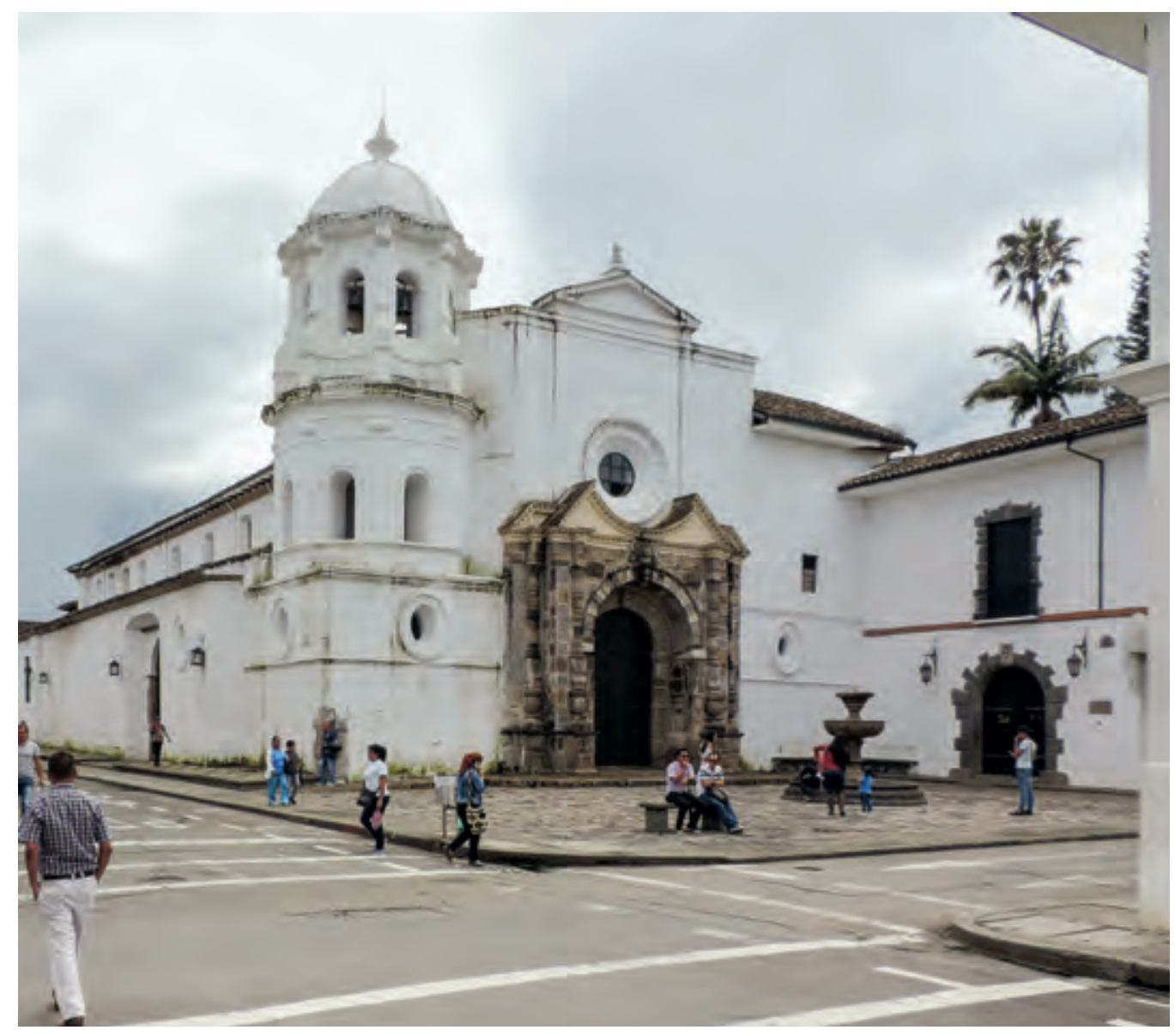

Imagen 1. Iglesia de Santo Domingo (Popayán).

Mariquita, hoy departamento del Tolima, y educado en el colegio San Bartolomé de Santafé, estuvo en España en momentos en que se adelantaron estos procesos de transformación. Así, a partir de la expulsión de los jesuitas del virreinato de la Nueva Granada, a él le fue encomendada la labor de llevar a cabo el proceso de transformación del sistema educativo. El nuevo sistema en la educación desplazó el modelo escolástico manejado por los colegios religiosos por las cátedras de matemáticas, filosofía y ciencias, entre lo que se destacó enseñar el modelo de Copérnico.

La Arquitectura no fue ajena a este tipo de reformas, y en España, a partir del reinado de Carlos III, el estilo barroco se mantuvo en gran medida por la influencia italiana en la península, a diferencia de Inglaterra y Francia, en donde el neoclásico era el estilo que se imponía en la arquitectura y el arte.

Este neoclásico resaltó los órdenes clásicos griegos y romanos, trabajados por Palladio y Vignola. Si bien el neoclásico se extendió en el resto de Europa, en España y sus colonias de ultramar, el barroco tuvo diversas manifestaciones con la influencia de la familia de arquitectos, canteros y artesanos Churriguera, que tenían el monopolio en la construcción de obras encargadas por la corona española, con lo cual lograban hacer su propia versión del estilo barroco, estilo llamado churrigueresco.

\section{El estilo churrigueresco o llamado también barroco} español se enriqueció en América con estilos indígenas y africanos, es decir, nació un barroco mestizo, cuya mayor influencia se vio reflejada en las iglesias construidas en el virreinato de Nueva España, virreinato del Perú y en menor medida en el virreinato de la Nueva Granada.

Es importante destacar que durante el tiempo en que Caldas permaneció en Popayán, su ciudad natal, estuvo trabajando como ayudante del arquitecto Andrés Marcelino Pérez de Arroyo y Valencia en algunos proyectos del presbítero, más exactamente en el diseño del atrio y púlpito de la iglesia de Santo Domingo.

La Arquitectura no era una disciplina ajena al joven Caldas, dados sus pasos de aprendizaje con el arquitecto 


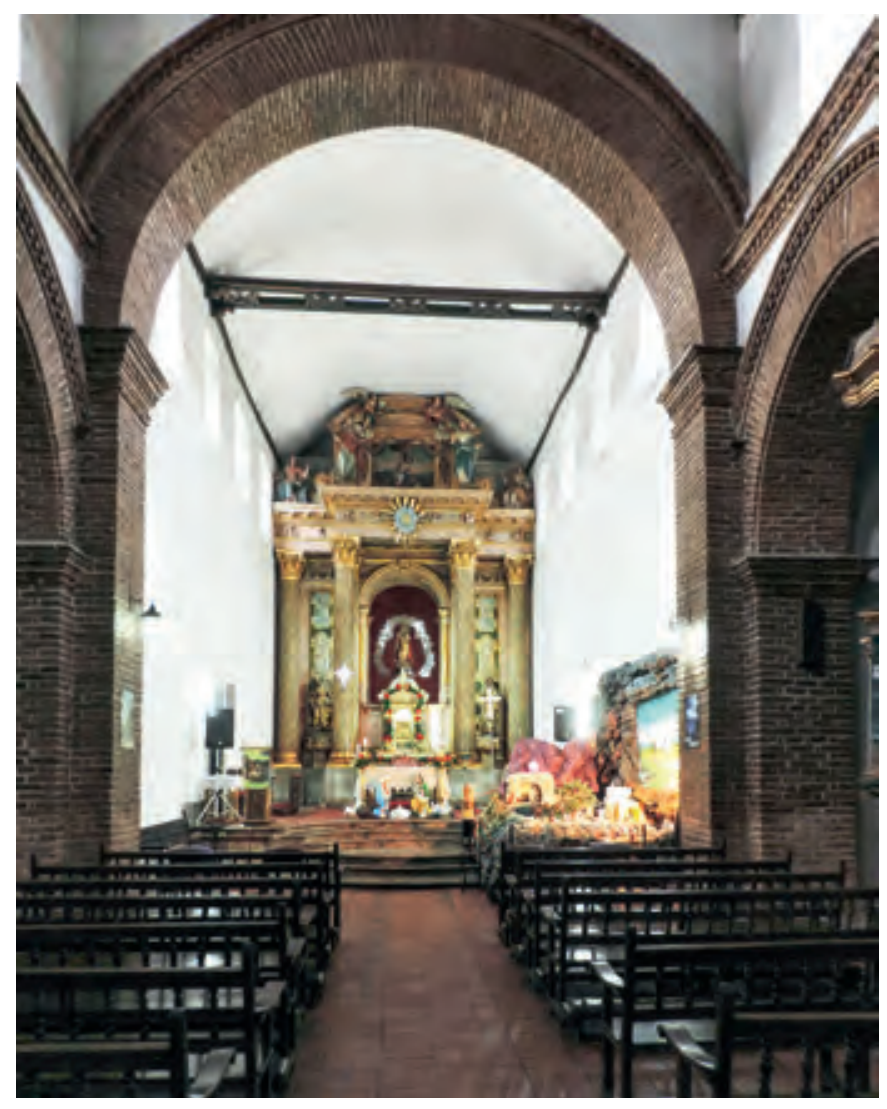

Imagen 2. Atrio y púlpito de Santo Domingo (Popayán). Fotografía: Jesús Antonio Villalobos.

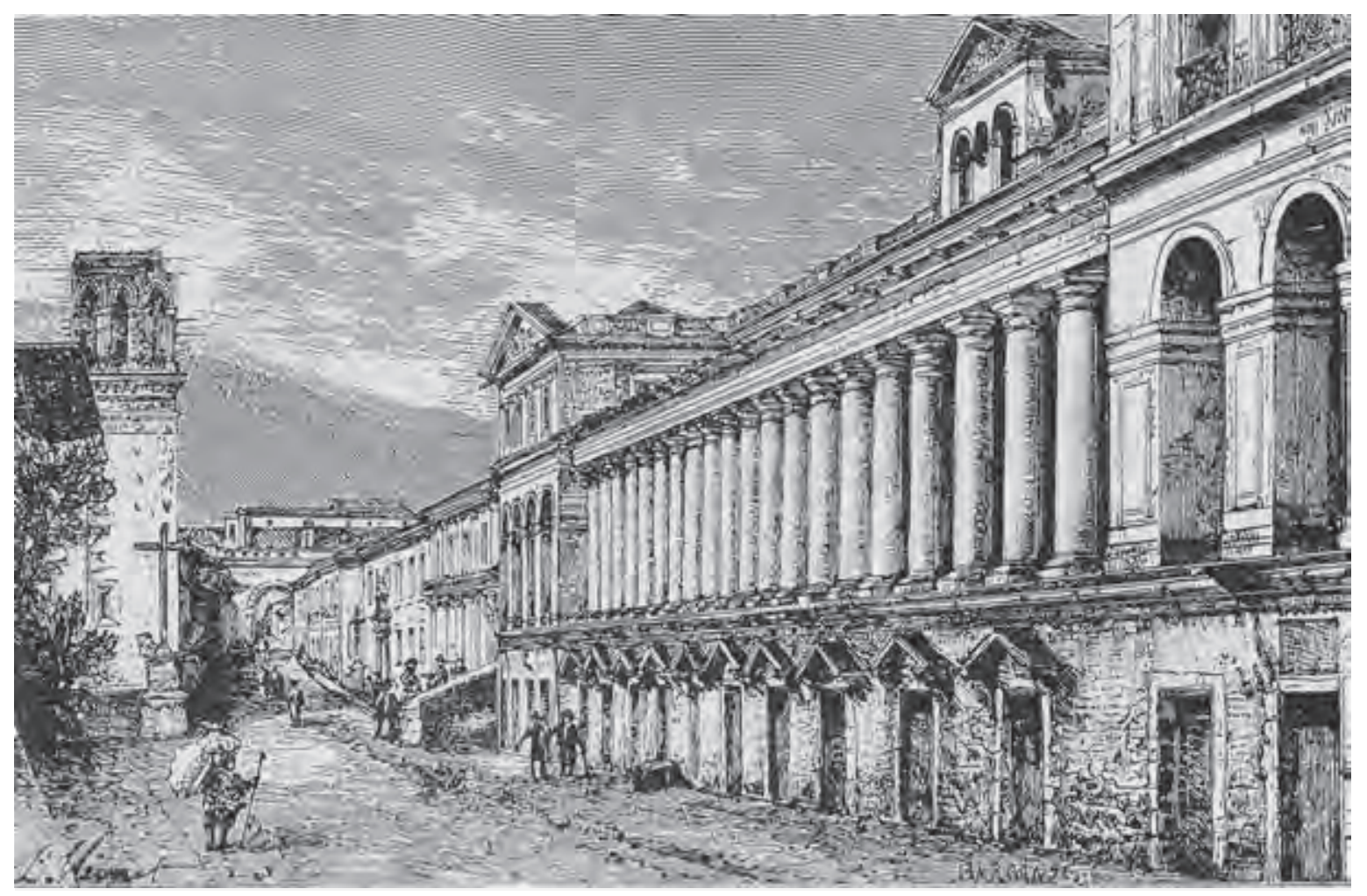

Imagen 3. Palacio de la Audiencia de Quito o Palacio de Gobierno. Fuente: Colciencias-Forum. Caldas 1768-1816. Molinos Velásquez Editores. (p. 149). 
Pérez de Arroyo, con quien logró adquirir el conocimiento de los órdenes clásicos de Vignola y Palladio, lo que le dio la experiencia necesaria para el juzgamiento de la arquitectura de su época. (Arango, 1993, p. 73).

La evidencia estudiada permite asegurar también que Francisco José de Caldas ejerció la arquitectura. Esta disciplina fue objeto de sus inquietudes intelectuales y para nada ajena a sus intereses. Los escritos y correspondencia que Caldas mantuvo con sus amigos y allegados permiten asegurar que él se encontraba familiarizado con el lenguaje de la arquitectura de su época. Por eso, no solo logró cultivar con fluidez y pasión la disciplina de la arquitectura, sino construir algunos de sus proyectos.

Igualmente, es importante destacar la habilidad constructiva ejecutada después de 1810 como parte del cuerpo de ingenieros militares en cada uno de los ejércitos de que hizo parte. Se sabe que en 1813 enseñó Arquitectura en el curso militar del cuerpo de ingenieros en la República de Antioquia y que dirigió obras de construcción de fortalezas, trazado de caminos, construcción de fábricas de pólvora y de hornos de fundición de cañones, entre otros.

\section{Descripciones de la arquitectura en la Presidencia de Quito}

Mientras Caldas permaneció en la Presidencia de Quito, en varias ocasiones le escribió a su amigo Santiago Pérez de Arroyo y Valencia, quien vivía en Santafé, apreciaciones de la arquitectura de esta ciudad, como lo refleja una carta del 21 de octubre de 1801, en la cual se refiere a la biblioteca pública de la siguiente forma.

La biblioteca pública es la que fue de los Jesuitas, y es preciso confesar que es pieza magníficamente adornada. (...) y corre sobre él una balaustrada para impedir que caiga el que ande por arriba. Sobre los resaltes de este pedestal y arrimadas a las pilastras de la balaustrada hay estatuas como de tres cuartas de alto, todas alusivas a las ciencias de que tratan los libros del estante a que pertenecen ${ }^{1}$

\footnotetext{
1 Carta n.o 46 que en su nota n.o 12 dice lo siguiente: “En la actualidad la Biblioteca de Quito funciona en este mismo lugar, y el recinto se conserva tal como Caldas lo describe. Caldas encontró allí obras de Jorge Luis Leclerc, Conde de Buffon, Pedro Luis Moreau de Maupertius, César Francisco Cassini, Renato Antonio Ferchault de Réamur, Henry Baker, Benjamín Thomson, conde de Rumford y las citas alegóricas a Nicolás Copérnico y Tycho Brabe.
}

(Academia Colombiana de Ciencias Exactas, Físicas y Naturales, 1978, p. 113).

Igualmente, se refiere a la arquitectura de la ciudad describiendo la plaza mayor en la Presidencia de Quito, el palacio presidencial, la audiencia y el palacio de los arzobispos, la catedral y el colegio de los jesuitas.

Todo este terreno está cercado de un buen edificio, uniforme, de un alto competente, y en el que tiene que observar un ojo inteligente; se elevan de trecho en trecho pilastras pareadas y proporcionalmente resaltadas con sus bazas y capiteles dóricos de gusto; sobre ellas corre su correspondiente cornisamento; cuyo friso carece de triglifos; sobre el vivo de las pilastras del cuerpo inferior se elevan otras tantas hasta la cornisa superior, del mismo orden; en los claros inferiores o interpilastros - permítame usted esta voz - hay ventanas con sus jambas correspondientes, y lo mismo en el superior (...).

(...) es en arco y correspondiente al orden; da entrada al primer claustro que hoy sirve de universidad; este es espacioso, compuesto de columnas dóricas, y sobre ellas una arcada con su cornisita a la imposta ${ }^{2}$. (Academia Colombiana de Ciencias Exactas, Físicas y Naturales, 1978, p. 120).

A partir del análisis realizado en las construcciones en la Presidencia de Quito, se identifica que Caldas dominó el lenguaje de los órdenes clásicos y la descripción en detalle, así como que se encontraba familiarizado con los estilos y denominación de los ornamentos descritos en la catedral y en el colegio de los jesuitas.

\section{Descripciones de la arquitectura Inca en Otavalo y Ambato, Presidencia de Quito}

A partir de su largos y penosos recorridos realizados en la Presidencia de Quito, el 7 de noviembre de 1802, a su salida de la ciudad de Ibarra y posterior llegada a Otavalo, en carta dirigida a José Celestino Mutis, Caldas realizó un detallado informe de su recorrido, haciendo énfasis en el lago San Pablo, en donde se encontraban algunos vestigios de arquitectura hecha por los incas, situación que no pasó desapercibida para Caldas, y de la cual dice:

2 Carta n.․ 48, pp. 120-122, igualmente en la carta n. 73 , pp. 199-202. En el archivo epistolar de Mutis, titulado Cartas al sabio Mutis, se encuentra esta carta, pp. 127-134. 
Remito a usted un monumento de los antiguos peruanos que he hallado en las orillas del lago de San Pablo, y que creo se ha escapado a las investigaciones de Ulloa (...).

(...) Es un edificio circular, de piedra bruta, labrada únicamente por el frente, y unida sólidamente con una argamasa en que no ha entrado cal. Está en gran parte arruinado no por los temblores, ni por el tiempo, sino por la mano bárbara del Cura de Otavalo. (Mariano Jácome) Este eclesiástico ha creído útil deshacer este monumento precioso, capaz por sí solo de darnos luces sobre la arquitectura, y sobre los ritos de este pueblo (...).

(...) Tiene 46 pies del rey de diámetro interior; el grueso de muro 4 pies, la altura 14 pies. No existe sino una sola puerta, y los indios me han asegurado había otra diametralmente opuesta, y en efecto, hay una abertura en este lado. Véase el plano. (plano que no ha sido hallado hasta el momento) Seguramente conocieron el plomo, pues lo está la parte que existe. Parece que no llegaron a conocer el arte de las bóvedas o cañones, pues aun cuando daban esta figura a sus puertas no colocaban las piedras en la dirección del radio, sino que buscaban las piedras chatas y largas, enlazándolas del modo que manifiesta el diseño, y llenando las cavidades que dejaban con la mezcla de que he hablado. Todo lo que va de negro en el perfil de la puerta, es lo que ocupa la argamasa ${ }^{3}$. (Academia Colombiana de Ciencias Exactas, Físicas y Naturales, 1978, p. 199).

En su memoria titulada Viaje al corazón de Barnuevo (Universidad Nacional de Colombia, 1966, p. 455) se hace referencia a su viaje por el sur de la Presidencia de Quito, en mayo de 1804, especialmente por la población de Ambato, dado que habla del decadente estado imperfecto de su arquitectura.

Sus calles, alineadas y agradables, tienen en lo principal dos series de sauces de la especie piramidal, que recuerdan la idea de una alameda en el centro

\footnotetext{
$3 \quad$ Carta 73, páginas 199 a 202. En el archivo epistolar de Mutis, titulado Cartas al sabio Mutis, se encuentra esta carta, páginas 127 a 134. Nota 4: "El original del dibujo se encuentra en el Jardín Botánico de Madrid. Mendoza lo reprodujo en su obra sobre la Expedición Botánica, pero con las leyendas levantadas en imprenta. Alude a Antonio Ulloa". En esta carta, según la nota 16, Caldas introduce el primer microscopio a Ecuador a partir de los instrumentos enviados por Mutis desde Santafé.
}

de la población. La entrada está adornada con una plazoleta o plataforma con algunos adornos no del mejor gusto y que hacen conocer a primera vista el estado imperfecto y decadente de la arquitectura. (Universidad Nacional de Colombia, 1966, p. 455).

En ese mismo viaje, en cercanías de la población de Ambato, se encontró con las ruinas de algunas edificaciones pertenecientes a construcciones hechas por los Incas. Caldas, de manera equivocada, se refiere a ellas como si fuesen un palacio, pero en nota incluida por el autor de las cartas a Caldas se hace la corrección cuando se dice que estas no son palacios, "(...) sino casas de hospederías para los pasajeros, que los incas habían hecho construir en estos parajes elevados." (Universidad Nacional de Colombia, 1966, p. 473).

Sin embargo, lo interesante es la apreciación que Caldas hace de estas construcciones describiendo su sistema constructivo y la arquitectura, y se destaca su admiración por este complejo en ruinas en particular, "Todo es de piedras sin labrar y bien diferentes del de Atuncañar, de que hablaremos bien pronto. Es de admirar cómo estos Emperadores dejasen los bellos sitios y la temperatura de Cuenca por estos lugares tristes, nebulosos y helados, para erigir edificios tan grandes y suntuosos." (Universidad Nacional de Colombia, 1966, p. 473).

Se ha mencionado que Juan de Ulloa, en su viaje con Godin y la Condamie, realizó algunos dibujos de las construcciones de las que Caldas hace mención, pero se sabe que Ulloa no era un experto en el arte del dibujo, lo que hace difícil comparar estas imágenes con las descripciones de Caldas.

El barón Von Humboldt en su recorrido por el sur de la Presidencia de Quito realizó levantamientos y descripciones de lo que denominó La Casa del Inca, en su libro Mi Viaje por el camino del Inca (1801-1802), en donde el notable científico ratifica las limitaciones de los dibujos de Jorge Juan haciendo la siguiente apreciación:

(...) entre ellas, una de las más celebres y mejor conservadas es la de Callo o Caio, que don Jorge Juan, [don Antonio] Ulloa y La Condamine, en sus viajes al Perú, describen, aunque imperfectamente, siendo tan poco exacto el dibujo con que Ulloa ha pretendido representar el plano de la casa del Inca, que casi pudiera creerse puramente imaginario. (Von Humboldt, 2004, p. 95). 
Continuando con las apreciaciones de Caldas acerca de La Casa del Inca, como la denomina el Barón Von Humboldt, se afirma que está invadida por un lugareño que le ha hecho mucho daño a su arquitectura, y que es difícil hacerse una idea de los patios y salones, debido a que todo está casi en ruinas, pero se puede destacar el torreón a partir de una figura elíptica de 110 pies de rey en dirección este a oeste en su mayor extensión y de 38 varas sobre el meridiano y que su altura perpendicular es de 16 varas. Continúa describiendo el lugar de la siguiente forma:

Todo este gran zócalo está cubierto o revestido exteriormente de un muro de piedras perfectamente cortadas en paralelepípedos de igual altura y de largo diferente, formando exteriormente una especie de almohadillado. Hoy tendrían trabajo los canteros armados del fierro y del acero en ejecutar otro tanto en igual grado de perfección. La arquitectura está tan atrasada hoy, que podemos decir que los indios en el seno de su barbarie hacían grandes ventajas a nuestros arquitectos. Once hileras de estas piedras se cuentan desde el suelo hasta su parte más alta.

(...) Sobre este zócalo y en el centro de la elipse se levanta un edificio cuadrado, dividido en dos por un muro que corre sobre el eje o diámetro menor, sin comunicación entre sí. Por el Este y Occidente tienen sus puertas, que comunican con las mitades de la elipse respectivas. Se notan muchas alacenas y piedras cilíndricas que sobresalen perpendiculares al muro. Es de la misma piedra y de la misma forma que la que hemos referido hasta la mitad de su altura, y de lodo mezclado con cascajo de aquí arriba. (Universidad Nacional de Colombia, 1966, p. 474).

Al final, Caldas se refiere a este emplazamiento Inca de gran magnitud, admirado por su torreón y bautizado así por Ulloa años antes, a pesar de su estado ya entrado en ruinas, "el lugar es uno de los más bellos que hemos visto en todo nuestro viaje. Véase el diseño de esa silla". 4 (Universidad Nacional de Colombia, 1966, p. 475).

\footnotetext{
4 El documento está acompañado de una nota hecha por el editor, en donde se menciona: "Desgraciadamente se han perdido todos 105 diseños y perfiles que acompañaban la relación de este viaje, y aun los nombres propios deben estar en mucha parte alterados por el copista. (Esta nota aparece en el libro del señor Acosta. Fue sin duda omitida por error de imprenta la inicial de dicho señor, que aparece en las demás notas). (R. P.)."
}

Durante su viaje desde la Presidencia de Quito hasta la ciudad de Santafé, en marzo de 1805, él se refiere a las descripciones realizadas por Ulloa, afirmando que este ignorante de la arquitectura no ha sabido describir los templos de la ciudad de Quito.

Esta afirmación hecha por el mismo Caldas, da a entender que él se considera con la autoridad para realizar juicios y apreciaciones sobre la arquitectura, asimismo, realiza descripciones de las casas, en su mayoría de la ciudad de Quito, afirmando que gran parte de ellas son de adobe y techos de maguey. A él le llaman especialmente su atención los muros, pisos y demás elementos que las adornan, y critica el mal gusto para su decoración. ${ }^{5}$ (Universidad Nacional de Colombia, 1966, p. 519).

Igualmente, se refiere al estado de las calles de la ciudad de Quito, resaltando su estrechez y su desaseo, porque la "plebe" deposita en sus calles el desperdicio; así las compara con las cloacas de la ciudad, y se lamenta de este estado:

(...) Esta es la suerte de la América, suerte triste que la mantiene a casi 300 años en el estado de abatimiento y de rudeza, a pesar de las intenciones paternales de nuestros reyes. El más bello y ventajoso establecimiento es el blanco del odio o del desprecio del sucesor.

La arquitectura no puede gloriarse ni de albañiles. Es cierto, tiene buenos trozos antiguos que manifiestan que los hubo inteligentes; pero no dejaron semilla. Todo posterior es un monstruo que deshonra al artista y al gusto de sus dueños (Universidad Nacional de Colombia, 1966, p. 519).

\section{Descripción del Observatorio Astronómico en Santafé de Bogotá}

Con la presencia borbónica desde la corte española en América, las influencias científicas y técnicas que esta impulsaba permitieron la llegada de personas cercanas a los círculos de poder que tenían conocimientos de matemáticas, geometría y levantamiento de planos, entre otros, como es el caso del capuchino Fray Domingo de Petrés, arquitecto de formación que llegó a Santafé hacia 1792, y que logró traer los últimos

$5 \quad$ Viaje de Quito a Popayán. Al parecer escrita entre su viaje del 28 de marzo de 1805 hasta su arribo a Popayán. 


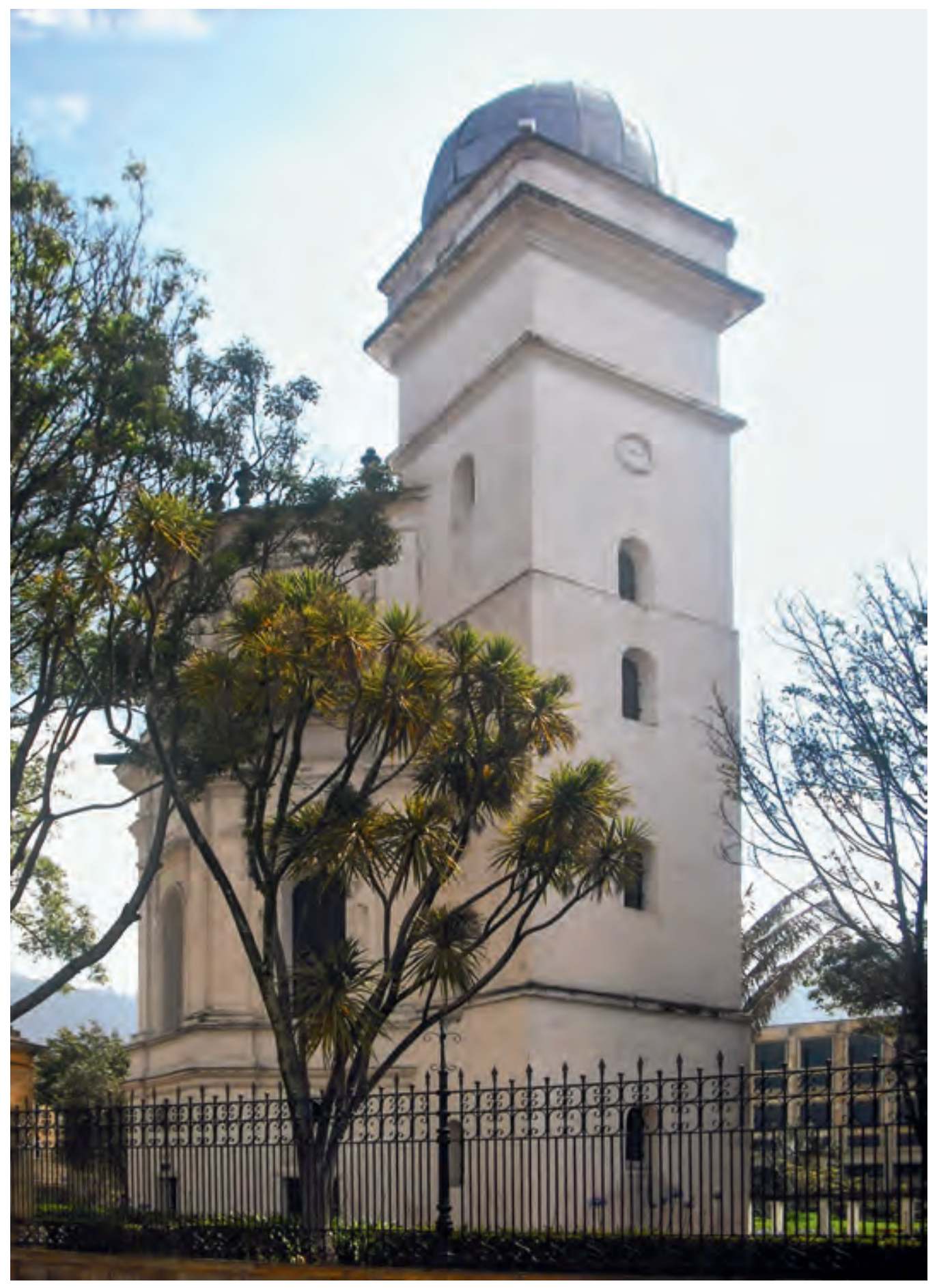

Imagen 4. Observatorio Astronómico de Santafé de Bogotá. Fotografía: Jesús Antonio Villalobos. 
conocimientos de los estilos arquitectónicos trabajados en Europa de fines del siglo XVIII.

Este arquitecto no utiliza el barroco español como recurso estilístico en los diseños y construcción de la catedral de Santafé y en el Observatorio Astronómico. Allí se identifica una clara influencia neoclásica que sería posteriormente, todo el siglo XIX, el estilo de la arquitectura de esta ciudad. Esta situación presente en la época de la independencia fue reforzada por los gobiernos de la nueva nación como una imagen de ruptura entre el Estado colonial y la naciente República. En la obra del Observatorio Astronómico, iniciada el 8 de mayo de 1802 y culminada el 20 de agosto de 1803, se identifica la influencia del Observatorio de Greenwich, en el Reino Unido, y de la Stellata, en la ciudad de París, en donde el arquitecto capuchino utilizó una clara geometría clásica para su concepción. (Cuéllar, 2012, p. 66).

El astrónomo Jorge Arias de Greiff menciona que fray Domingo de Petrés tomó como modelo la primera construcción del Observatorio de Greenwich y las torres extremas de la primera construcción del Observatorio de París. ${ }^{6}$ (Colciencias-Forum, 1994, p. 46).

Esta situación de haber tomado como modelos los observatorios europeos condujo a errores sustanciales en el diseño y disposición de espacios, ventanas y escaleras, que riñen con la lógica de un observatorio para lugares localizados cerca del ecuador, situación que Caldas menciona en su memoria, y que trata sobre la construcción del Observatorio Astronómico.7 (Colciencias-Forum, 1994, p. 40).

En Santafé, Francisco José de Caldas, después de su reconocimiento casi totalmente de la Presidencia de Quito, entre 1801 y 1805, recibe del director de la Real Expedición Botánica de la Nueva Granada, José Celestino

En la nota n.o 38 describe que la Cámara Stellata, debida a Christopher Wren, data de 1675; la construcción inicial de París remata en dos torres octogonales en sus extremos norte y sur, y el arquitecto fue Claude Perrault.

$7 \quad$ Arias de Greiff describe los errores a los que Caldas se refirió en relación a los salones que son parte del Observatorio en su disposición, los cuales son ubicados en lugares diferentes al ecuador: "(...) altas latitudes europeas para observar desde el interior, por las ventanas, la marcha de los de los cuerpos del sistema solar desde la salida del oriente, hasta la puesta por occidente, con su máxima altura o culminación, no en el cenit sino hacia el sur, momento en el que se veía en la ventana del costado. Pero no ocurre en las zonas ecuatoriales, donde estos astros culminan muy altos vecinos al cenit. Todavía más: Ni siquiera hay ventana al sur pues ese es el lugar de la escalera."
Mutis, la tarea de hacerse cargo del Real Observatorio Astronómico de la Nueva Granada como director titular.

Ante tal ofrecimiento por parte del sabio Mutis, Caldas pareció desilusionado, después de su memorable trabajo realizado en la Presidencia de Quito. Esperaba que le asignaran trabajo como botánico y no como astrónomo, porque así iba a quedar desligado de su trabajo memorable sobre las quinas de Loja. Todo esto se trató de una jugada maestra por parte del director de la Real Expedición Botánica de la Nueva Granada, José Celestino Mutis, ya que así no pondría en riesgo su negocio con las quinas.

En la misma carta dirigida al secretario del Virreinato y juez comisionado para los asuntos de la Real Expedición Botánica, Caldas realiza un juicioso resumen de su trabajo para la Real Expedición Botánica, en el cual destaca su trabajo inicial en la Provincia de Quito desde 1802 a 1805 . Alega que su principal trabajo ha sido como botánico y no como astrónomo.

No obstante, en la nota No. 3 de la carta se aclara que Mutis, si bien le encomendó la tarea de hacer trabajos botánicos en Quito, su idea era que Caldas estuviese a cargo del observatorio Astronómico en Bogotá.

Caldas asume el cargo de director del Observatorio Astronómico y realiza una descripción del estado del mismo, la cual fue publicada posteriormente a su muerte con el título de Descripción del Observatorio Astronómico de Santa Fe de Bogotá situado en el jardín de la Real Expedición Botánica, descripción seguramente escrita para 1805, tiempo en que se desempeñó como director del Observatorio.

La descripción hecha por Caldas inicia describiendo la torre octogonal de 13 pies de rey de lado y una altura de 56 de altura. El diámetro, quitando el grueso de los muros es de 27 pies. Tiene tres cuerpos: el primero, de 14,5 pies de elevación de pilastrones toscanos pareados en los ángulos, sobre un zócalo que corre por todo el edificio. En los columnarios hay ventanas rectangulares, y en el que mira al Oriente está la puerta. La bóveda sostenida por este cuerpo forma el piso del salón principal. El segundo, de 26,5 pies, en orden dórico, en pilastras angulares como el primero. Dentro de ellas están las ventanas muy rasgadas, circulares por arriba, con recuadros y guardalluvias que las adornan. La bóveda superior es hemisférica, perforada en el centro y sostiene el último piso al descubierto. Un ático fingido corona todo el edificio y sirve al mismo tiempo de antepecho. 
El agujero de la segunda bóveda da paso a un rayo de luz que va a pintar la imagen del sol sobre el pavimento del salón, en que se ha tirado una línea meridiana y forma un gnomon de 37 pies y 7 pulgadas de elevación.

En relación a la obra como Observatorio Astronómico, Caldas tiene sus reparos en relación a la ubicación y capacidad del mismo edificio para realizar observaciones tal como ya se afirmó.

Luego es preciso decir que Caldas no participó en el diseño y construcción del Observatorio Astronómico de Santafé, a diferencia de lo que se insinúa en el documento de Silvia Arango, en el entendido de que cuando se construye el observatorio, Caldas se encontraba en Quito, pero es cierto que él sabía de esta obra por intermedio de la comunicación epistolar con Mutis, quien lo tenía al tanto de esta situación.

Igualmente, Arias de Greiff, a partir de su conocimiento como astrónomo, afirma que la obra hecha por el arquitecto Fray Domingo de Petrés, con dinero de las quinas que comercializaba Mutis, se constituyó para la época en un observatorio obsoleto que respondía a la tecnología constructiva para este tipo de edificios en el siglo XVI, “(...) erigió un observatorio obsoleto, diez años después de que en Madrid y San Fernando se habían construido modernos edificios, no de la tipología del siglo XVI, pero si ya de la que caracteriza las construcciones del siglo XIX." (Colciencias-Forum, 1994, p.40).

Finalmente, mientras estuvo a cargo como director de la Real Expedición Botánica José Celestino Mutis, Caldas se desempeñó de buena forma como director del Observatorio Astronómico, pero era clara su insatisfacción, tal vez albergando la esperanza de que una vez Mutis dejara su cargo como director de la Real Expedición botánica, este cargo recaería en él, situación que no sucedió tal como se mencionó anteriormente.

Restituido a esta capital, se me hicieron las ofertas más lisonjeras y se me entregó como un objeto secundario el Observatorio Astronómico que se acababa de erigir, con los instrumentos que el Rey había dado a nuestra Expedición. Estos no habían salido de las cajas que los contenían, y yo los estrené lo mismo que el edificio. En este he tirado una exacta meridiana, que me hizo conocer la poca inteligencia con que se habían formado los planos y puesto los cimientos; he notado todas las faltas y defectos que contiene este establecimiento, y he verificado una serie escrupulosa de todas las observaciones de que es capaz el edificio y los instrumentos. (Academia Colombiana de Ciencias Exactas, Físicas y Naturales, 1978, p.274).

\section{Trabajos realizados como ingeniero militar}

A partir de su huida de Santafé, después de la batalla perdida contra Antonio Nariño en el intento de tomar por las armas esta capital del Nuevo Reino de Granada y de defender la causa federalista liderada por su primo Camilo Torres, Caldas en su viaje a Cartagena pasa por la Provincia de Antioquia, lugar que el presidente dictador Juan del Corral por decreto había decidido mejorar mediante el Plan y nuevo arreglo general de la fuerza armada permanente para la Republica de Antioquia. El plan contemplaba tareas como la organización de un cuerpo de ingenieros y la maestranza de artillería, al igual que la realización de trabajos de fortificación en Caramanta, Arquía, Cana y Bufú, sobre el río Cauca. ${ }^{8}$ (Academia Colombiana de Ciencias Exactas, Físicas y Naturales, 1978, p.346).

Estos trabajos fueron encomendados a Caldas, quien después de realizar las fortificaciones antes mencionadas, regresa para organizar la Escuela Militar de Cuerpos Facultativos, proyecto que ve la luz en el mes de agosto de 1814, en la ciudad de Medellín, pero en carta al presidente Juan del Corral, Caldas le recuerda que los planos enviados se destacan por la información propia de la ingeniería, y que no se ocupe de los dibujos, ya que estos están realizados a partir de la premura: "Vea Vuestra Excelencia las líneas fundamentales, vea Vuestra Excelencia al ingeniero y no al pintor."9 (Academia Colombiana de Ciencias Exactas, Físicas y Naturales, 1978, p.346).

En el discurso preliminar como coronel del Ejército de la República de Antioquia, Caldas da por inaugurado el Primer Curso Militar del Cuerpo

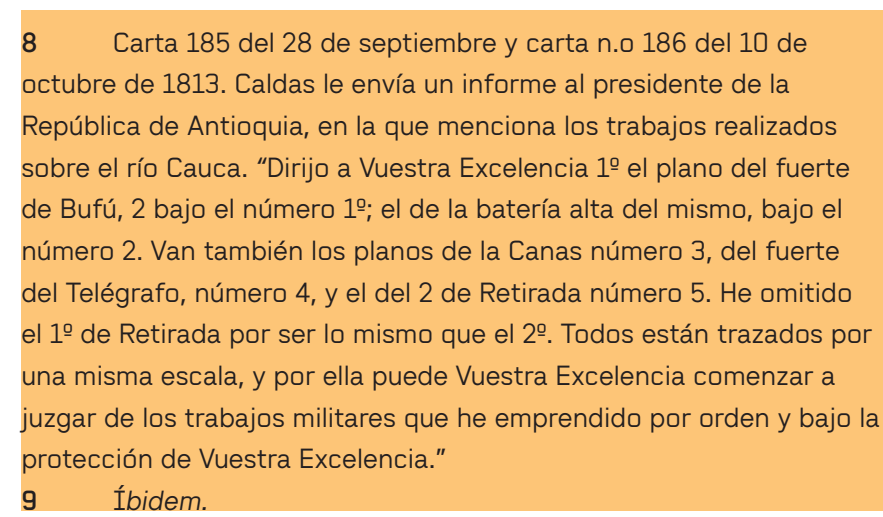




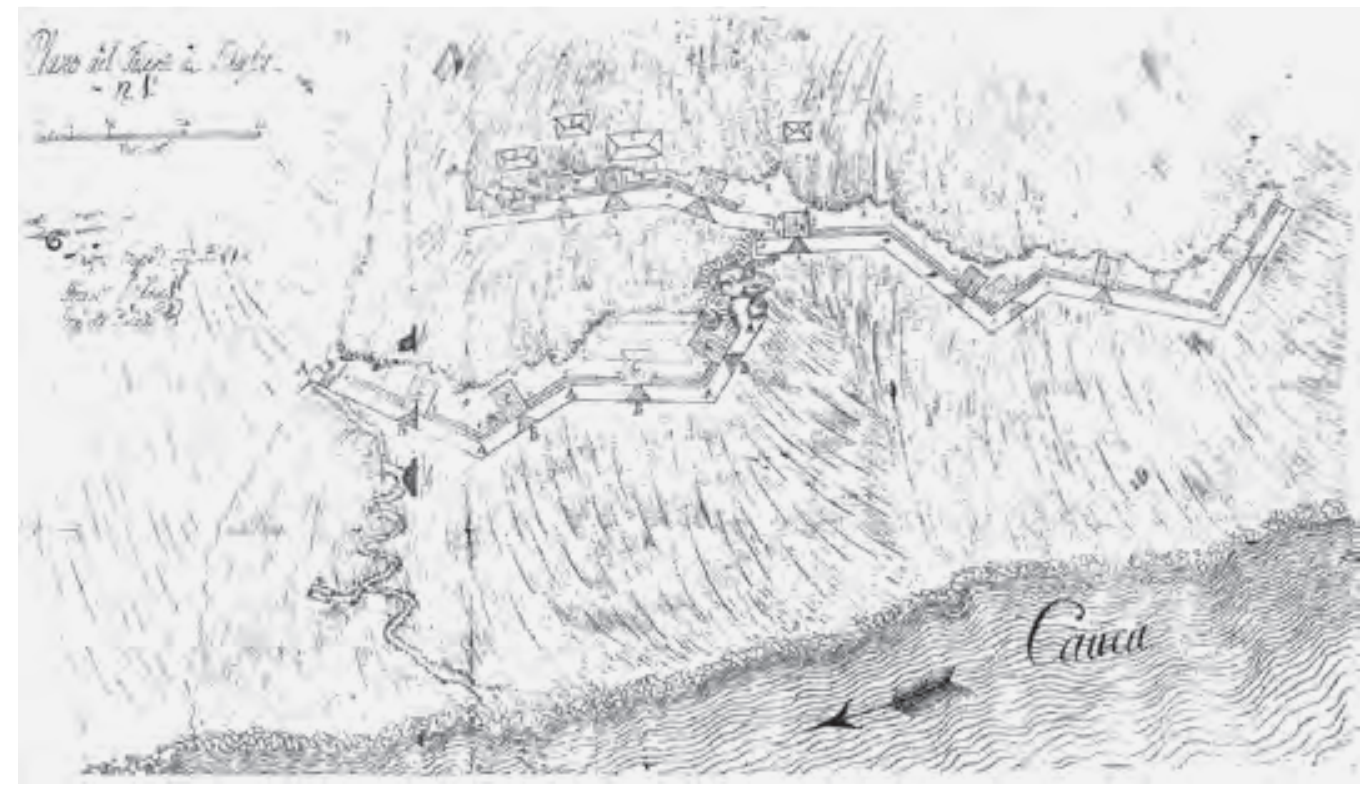

Imagen 5. Plano del Fuerte de Bufú. Imagen tomada del artículo: Francisco José de Caldas y la Geografía Militar en la Provincia de Antioquia. (1813-1815). Iván Felipe Suárez Lozano. Revista Apuntes 26(1), p. 50. Pontificia Universidad Javeriana.

de Ingenieros, incorporando la enseñanza de la Arquitectura como materia importante para la formación de estos cadetes. (Universidad Nacional de Colombia, 1966, p.55).

El primer tratado será la Arquitectura militar o Fortificación. Aquí aprenderéis a fortificar plazas y a cubrir la campiña; a atacar a un enemigo atrincherado por medio de muros robustos; aquí veréis las sublimes ideas de Vauban, Cohorn, Deville, Turpin... para pelear y vencer a enemigos numerosos con un puñado de hombres. (...).

\section{(...) El tercero será la Arquitectura hidráulica.} Canales, acueductos, molinos, esclusas, bombas, norias, toda la fuerza de las aguas aprovechadas será el objeto de esta tercera parte. (...).

\section{(...) En fin, el sexto estará consagrado a la Arqui-} tectura civil. Ella levanta templos al Señor, palacios a la autoridad pública, casas risueñas al ciudadano, construye puentes, calzadas, caminos para la utilidad general, y llena la vida de bienes y comodidades. Todos estos conocimientos son útiles y necesarios a un militar que debe despreciar esas sutilezas estériles y solo ocuparse del hombre, porque la ciencia de sus necesidades y los medios de remediarlas es lo que hace verdaderamente sabios. (Universidad Nacional de Colombia, 1966, p. 55).
Finalmente, antes de partir de nuevo para Santafé, en septiembre de 1815 , deja la construcción de una fábrica de nitro, la casa de la moneda y una fábrica de fusiles en la ciudad de Rionegro. (Riaño, 1994, p. 150).

\section{Conclusiones}

Es importante destacar que el tiempo de Caldas es una época de influencias ilustradas, en gran medida auspiciadas por la misma corte española, a partir de los cambios introducidos por el reinado de Carlos III en España. Luego las colonias de ultramar se consideraron el objetivo de exploradores, científicos e investigadores, quienes veían que en estas tierras todavía se escondían grandes riquezas que era posible descubrir y comercializar.

Es igualmente importante mencionar que Caldas en su etapa de formación se encontró en medio de profundos cambios, por el modelo educativo impulsado por la corona española en esa época, modelo que se encontraba influenciado por la Ilustración que invadía los recintos científicos y las cortes de Europa de mediados del siglo XVIII.

Es sabido también que en la época en que Caldas estudió en el Colegio Mayor de Nuestra Señora del Rosario la licenciatura de abogado, se quejó del modelo 
escolástico de la enseñanza, añorando materias como la aritmética, geometría, trigonometría, algebra y física experimental, que ya eran objeto del modelo ilustrado en toda Europa.

Se destaca que la formación matemática, filosófica y científica incipiente en Caldas fue producto de su maestro José Félix Restrepo, que siendo su maestro en la ciudad de Popayán, muy seguramente ya entendía los cambios necesarios en los contenidos de la enseñanza. $^{10}$ (Academia Colombiana de Ciencias Exactas, Físicas y Naturales, 1978, p. 99).

Por las razones anteriores y a partir de la evidencia recabada se logró identificar que Francisco José de Caldas diseñó, proyectó, construyó y adelantó trabajos de apreciación y crítica en el campo de la arquitectura, a partir de los conocimientos adquiridos con su maestro Marcelino Pérez Arroyo y Valencia. Luego es a partir de la experiencia empírica y como discípulo de este presbítero payanés que Caldas logró cimentar sus conocimientos en arquitectura.

El trabajo realizado en la Presidencia de Quito trascendió la simple descripción de sus edificaciones. Allí Caldas realizó un análisis y crítica a la arquitectura, a partir de identificar la falta de pericia y talento de los albañiles y canteros en la realización de estas obras. Igualmente, se lamentó de la poca suerte que tuvo la arquitectura en esta parte de América, lo que identificó como una clara manifestación de desaprobación al estilo barroco, estilo que no era de su agrado.

Todo lo contrario sucede con las edificaciones en ruinas realizadas por los Incas, en las que Caldas reconoció el talento de sus constructores al decir que los Incas aventajaron a los arquitectos españoles: "La arquitectura está tan atrasada hoy, que podemos decir que los indios en el seno de su barbarie hacían grandes ventajas a nuestros arquitectos." (Universidad Nacional de Colombia, 1966, p. 474).

En este mismo sentido, cuando asumió el cargo de director del Real Observatorio Astronómico de Santafé, identificó claramente que el edificio era obsoleto. Estableció que su proyectista y constructor, el presbítero Fray Domingo de Petrés, al estar influenciado

10 Carta No. 41 del 5 de agosto de 1801. Página No. 99. Igualmente, en: Herrera Restrepo, Daniel. José Félix Restrepo, Maestro de Caldas. En Colciencias-Forum. 1994. Caldas 1768-1816. Francisco José de Caldas. Molinos Velásquez Editores. pp. 23-35. por los observatorios de Greenwich y de París, ignoró las condiciones de localización de un edificio de estas características, dado que en el ecuador no eran iguales a las del hemisferio norte.

Años después su experiencia y conocimiento en los campos de la arquitectura y la construcción fueron de gran provecho para la causa de la independencia, siendo este el hecho más significativo de la época en la vida de Caldas, así como haber logrado incluir por primera vez en la enseñanza la arquitectura y la construcción en una institución pública.

Si bien es claro que la pasión de Francisco José de Caldas estuvo marcada por la botánica y la astronomía, como en la física atmosférica, disciplinas que ejerció en todos sus viajes por el Nuevo Reino de Granada, también es claro que, a partir de su obra epistolar, la arquitectura hizo parte de sus pasiones, y lo acompañó paralelamente en su trabajo científico realizado en el virreinato.

Finalmente, su aporte como prócer a la independencia se puede entender desde dos puntos de vista: el primer aporte se dio por el hecho de dedicar su vida al redescubrimiento de las riquezas naturales en el Nuevo Reino de Granada, lugar que destaca su posición privilegiada, la zona ecuatorial, y situación que para los criollos ilustrados fue fundamental para acoger la causa de la independencia; y el segundo aporte está en haber incluido por primera vez en el pénsum académico de una escuela de formación de ingenieros militares el estudio de la Arquitectura, convirtiendo esta actividad en la síntesis que permite construir materialmente una nueva nación.

\section{Referencias}

Academia Colombiana de Ciencias Exactas, Físicas y Naturales. (1978). Cartas de Caldas. Bogotá, Colombia.

Arango, S. (1989). Capítulo II Arquitectura Colonial en Historia de la Arquitectura en Colombia.Universidad Nacional de Colombia. Bogotá: Editorial Facultad de Artes.

Arias de Greiff, J. (1994). Caldas: Inquietudes, Proyectos y Tragedias. En: Colciencias-Forum. Caldas 1768-1816. Francisco Joseph de Caldas y Thenorio. Molinos Velásquez Editores. 
Cuéllar, M. (2012). El primer arquitecto en la Nueva Granada y sus ideas ilustradas en: Fray Domingo de Petrés en el Nuevo Reino de Granada. Alcaldía Mayor de Bogotá [en línea] Disponible en: https://issuu.com/patrimoniobogota/docs/ fray_domingo_de_petr_s_en_el_nuevo

Riaño, C. (1994). Coronel Francisco José de Caldas.

En: Colciencias-Forum. Caldas 1768-1816.

Suárez Lozano, I. F. (2013). Francisco José de Caldas y la Geografía Militar en la Provincia de Antioquia.

(1813-1815). Revista Apuntes, 26(1).Bogotá: Pontificia Universidad Javeriana.

Universidad Nacional de Colombia. (1966). Viaje al corazón de Barnuevo. Obras completas de Francisco José de Caldas, publicadas por la Universidad Nacional de Colombia como homenaje, con motivo del sesquicentenario de su muerte. Bogotá: Imprenta Nacional.

Von Humboldt, A. (2004). Mi viaje por el camino del inca (1801-1802). Antología. Edición y prólogo por David Yudilevich. Santiago de Chile: Editora Universitaria. 